\title{
MAGNETIC RESONANCE IMAGING OF IRON OXIDE LABELLED STEM CELLS: APPLICATIONS TO TISSUE ENGINEERING BASED REGENERATION OF THE INTERVERTEBRAL DISC
}

\author{
K.J. Saldanha ${ }^{1,2, *}$, S.L. Piper $^{3,4}$, K.M. Ainslie ${ }^{5}$, T.A. Desai ${ }^{2,5}$, H.T. Kim³ ${ }^{3}$ S. Majumdar ${ }^{1,2}$ \\ ${ }^{1}$ MQIR, Department of Radiology, University of California, San Francisco \\ ${ }^{2} \mathrm{UC}$ Berkeley/UCSF Joint Graduate Program in Bioengineering \\ ${ }^{3}$ Dept. of Orthopaedic Surgery, University of California, San Francisco \\ ${ }^{4}$ University of California, San Francisco School of Medicine \\ ${ }^{5}$ Dept. of Physiology/Division of Bioengineering, University of California, San Francisco, USA
}

\begin{abstract}
Minimally-invasive monitoring of regeneration in diseased tissue is an important aspect of stem cell therapy. Magnetic resonance imaging (MRI) based tracking of cells labelled with ferumoxides has the potential for non-invasive in vivo detection and longitudinal assessment of implanted cells. Cells labelled with ferumoxides appear as hypointense regions on MR images and thus can be distinguished from the surroundings. Application of this methodology to intervertebral disc degeneration (IVD), and detection of labelled cells implanted into the disc for tissue regeneration was examined. Mesenchymal stem cells labelled with a ferumoxide contrast agent were imaged in vitro to quantitatively characterize the signal intensity loss using MRI relaxation parameters $\left(\mathrm{T}_{1}, \mathrm{~T}_{2}\right.$, and $\left.\mathrm{T}_{2}{ }^{*}\right)$. To determine whether labelled cells could be detected within scaffolds suitable for implantation, labelled cells were seeded within both natural and synthetic polymers and imaged using MRI. Labelled cells were loaded within poly(ethylene glycol) hydrogels and imaged in vitro using both MRI and confocal microscopy. Labelled cells were also loaded into fibrin gels, and detected ex vivo within rat IVDs using MRI. Lastly, the effect of ferumoxide labelling on cell viability was investigated. Quantitatively, labelled cells demonstrate the greatest signal intensity loss and contrast on $\mathrm{T}_{2}{ }^{*}$-weighted images. Labelled cells can be detected in both synthetic and natural polymers, and can be distinguished from the native tissue environment of the rat IVD. Finally, labelling does not significantly impair cell viability. Consequently, this technique shows promise as a potential method for in vivo longitudinal tracking of stem cell based regeneration of the IVD.
\end{abstract}

Keywords: Stem cell, iron oxide, intervertebral disc degeneration, magnetic resonance imaging, stem cell tracking, contrast agent, tissue engineering.

*Address for correspondence:

K.J. Saldanha

Box 2520, QB3 Building

$1700-4^{\text {th }}$ Street Suite 203

San Francisco, CA 94158, U.S.A.

E-mail: kjs@berkeley.edu

\section{Introduction}

Stem cell based tissue engineering therapies involve the administration of ex vivo manipulated stem cell populations with the purpose of repairing and regenerating damaged or diseased tissue. Recent studies have reported that stem cells have the potential to facilitate regeneration of a variety of tissues such as brain (Takagi et al., 2005), myocardium (Orlic et al., 2001, 2003), liver (Lagasse et al., 2000), and endothelium (Takahashi et al., 1999). Another potential application of stem cell therapy is in the treatment of intervertebral disc (IVD) degeneration, a condition that affects approximately 12 million Americans (Web ref. 1).

The biology of the IVD and the changes that occur with degeneration have been extensively reviewed (Beadle, 1931; Boos et al., 2002). Briefly, IVD anatomy consists of an inner core known as the nucleus pulposus, and a surrounding firm ring of tissue called the anulus fibrosus. During degeneration there are a variety of morphological changes that occur (Battie and Videman, 2006; Pfirrmann et al., 2001). For example, the distinction between nucleus pulposus and anulus fibrosus degrades, and the disc loses both height and hydration. Although clinically the link is poorly understood, IVD degeneration is postulated as a potential cause for chronic back pain (Urban and Winlove, 2007).

At the cellular level, the IVD consists of chondrocytes at the end-plates of the disc, chondrocyte-like cells in the nucleus pulposus, and fibroblast cells in the anulus fibrosus. The nucleus pulposus and anulus fibrosus contain abundant amounts of extracellular matrix (ECM) proteins, particularly collagens and proteoglycans (Leung et al., 2006). Since degradation of the ECM, in particular proteoglycans is known to be an early event in IVD degeneration current biological therapies are aimed at ECM restoration. In particular, a variety of studies have investigated mesenchymal stem cells (MSCs), a type of adult stem cell, as a means of regenerating the IVD. Rabbit MSC implantation resulted in an increase in proteoglycan content (Sakai et al., 2003; Zhang et al., 2005), partial restoration of disc height and hydration (Sakai et al., 2005, 2006), and differentiation into the chondrocyte/nucleus pulposus cell phenotype (Sakai et al., 2005).

To evaluate the effectiveness of such stem cell based therapies, it is necessary to monitor them once implanted within the body. In the case of the IVD, much of the previous work within rabbit and rat models of disc 
degeneration has relied on histological techniques for discontinuous assessment. Specifically, in order to detect the presence of cells within the region of interest at various time points, animals are usually sacrificed and stained specimen sections are viewed using microscopy (Crevensten et al., 2004; Sakai et al., 2006). While these techniques are effective in a research setting, they fundamentally preclude in vivo assessment and in vivo longitudinal tracking of therapy. As such, while stem cell therapies can be designed for minimally invasive implantation, the current methods of assessment are highly invasive and clearly clinically undesirable. For stem cell therapies aimed at repairing and regenerating the IVD to be clinically applicable, an effective technique for noninvasive in vivo detection and longitudinal tracking of transplanted cells is necessary.

An alternative method that can be used for monitoring implanted stem cells is to label the cells with a ferumoxide (FE) based contrast agent and track the cells using magnetic resonance imaging (MRI). This methodology would aid in the development and evaluation of stem cell based therapies for repair and regeneration (Arbab et al., 2004a; Bulte et al., 2001; Frank et al., 2003; Hoehn et al., 2002). With this technique, the contrast agent consisting of superparamagnetic iron oxide (SPIO) nanoparticles is taken up by the cell, increasing its magnetic susceptibility. As negative contrast agents, FEs weaken the MR signal intensity (SI), and thus appear darker in MR images. Signal intensity can be quantitatively characterized by $\mathrm{T}_{1}, \mathrm{~T}_{2}$, and $\mathrm{T}_{2} *$ relaxation time measurements, with a characteristic FE induced signal loss on $\mathrm{T}_{2}$ and $\mathrm{T}_{2}{ }^{*}$ weighted images (Frank et al., 2003; Kalish et al., 2003). However, FEs have a negative zeta potential, and thus are not efficiently taken up by stem cells and other nonphagocytic cells (Frank et al., 2003; Kalish et al., 2003). As such, a transfection agent is necessary to increase the efficiency of contrast agent uptake by the cell.

Arbab et al. have described the effective complexing of FEs with the transfection agent protamine sulphate (Pro) and labelling of a variety of cell types, including human MSCs (hMSCs) (Arbab et al., 2004b; Arbab et al., 2005). This FE-Pro complex allows for efficient cellular uptake of SPIO nanoparticles via endosomal incorporation, and subsequent MRI detection. A major advantage of the FEPro complex is that both FEs and Pro are approved for in vivo human use by the US Food and Drug Administration. As such, the transition of this methodology into the clinic would be relatively straightforward.

The purpose of this study was to investigate and further characterize FE-Pro labelling of hMSCs, and to explore the potential application of this novel methodology to detection of stem cells implanted into the disc for IVD regeneration. In addition to in vitro labelling of hMSC cultures, and quantitative characterization of SI loss on MR images (i.e. $\mathrm{T}_{1}, \mathrm{~T}_{2}$, and $\mathrm{T}_{2}{ }^{*}$ relaxation), the effects of labelling on stem cell viability were examined. In order to determine whether labelled cells could be detected within 3D scaffolds suitable for implantation, labelled cells were seeded within constructs and imaged using MRI. Since tissue engineering constructs are either synthetic or natural polymers (Elisseeff et al., 2006), this study investigated detection of labelled stem cells within these two types of matrices. For synthetic polymer investigation, labelled cells were seeded within biocompatible poly(ethylene glycol) (PEG) hydrogel plugs and imaged in vitro using both MRI and confocal microscopy. As a natural polymer, a fibrin gel was used to load labelled cells and implanted ex vivo within a rat model of IVD to demonstrate initial detection of transplanted cell populations via MRI. The results further validate the use of MRI to track FE-Pro labelled stem cells, and support the potential application of this methodology for monitoring transplanted stem cell populations throughout the IVD repair and regeneration process.

\section{Materials and Methods}

\section{Stem cells and cell culture}

Following procedures approved by the Institutional Review Board at the University of California, San Francisco CA, USA, bone marrow tissue was obtained from young adult male patients undergoing surgery for repair of femur fractures. Mononuclear cells were isolated by Percoll (Sigma Aldrich, St. Louis, MO, USA) gradient separation, and then plated in high glucose Dulbecco's Modified Eagle's Medium (DMEM) (Invitrogen, Carlsbad, CA, USA) with $10 \%$ foetal bovine serum (FBS) (Invitrogen) and 1\% penicillin/streptomycin (Invitrogen). After 24 hours, non-adherent cells were washed away. Adherent cells were maintained in the same media, expanded using standard culture conditions $\left(37^{\circ} \mathrm{C}\right.$ and $5 \% \mathrm{CO}_{2}$ ), and passaged $1: 4$ at $80-90 \%$ confluence. Cells were subcultivated using $0.25 \%$ trypsin with $1 \mathrm{mM}$ ethylenediamine- tetraacetic acid (EDTA) (Invitrogen). Cells used for labelling experiments were between passages 8-10 to avoid cell senescence.

\section{Cell labelling}

The FE-Pro labelling procedure was performed as described in Arbab et al. (Arbab et al., 2004b; Arbab et al., 2005). Briefly, the contrast agent Feridex IV (Berlex Laboratories, Wayne, NJ, USA) at a concentration of 100 $\mu \mathrm{g} / \mathrm{mL}$, and the transfection agent Protamine Sulphate (Pro; American Pharmaceutical Partner, Schaumburg, IL, USA) at a concentration of $4 \mu \mathrm{g} / \mathrm{mL}$ were added to DME serumfree medium and mixed for approximately 10 minutes. Following mixing, $12.5 \mathrm{~mL}$ of the labelling medium was added to an $80 \%$ confluent hMSC monolayer in a $150 \mathrm{~cm}^{2}$ cell culture flask. After 2 hours of incubation under standard culture conditions, an equal volume of complete DMEM was added to the cultures for a final FE concentration of $50 \mu \mathrm{g} / \mathrm{mL}$. Cell cultures were then incubated overnight.

Following labelling with FE-Pro, samples were washed with phosphate-buffered saline (PBS) containing heparin (Abraxis Bioscience, Los Angeles, CA, USA) at a concentration of 10 US Pharmacopeia (USP) units/mL. Heparin was used during washing because it competes with the ferumoxides for binding to protamine sulphate, thus working to dissolve cell surface bound FE-Pro particles (Arbab et al., 2004b) and deactivate extracellular FE-Pro. 
Of note, heparin is also approved by the Food and Drug administration and is used as an anticoagulant clinically. Cells were then trypsinized, collected, filtered through a $40 \mu \mathrm{m}$ mesh (BD Biosciences, Bedford, MA, USA), and counted for further experiments.

\section{FE-Pro hMSCs in synthetic hydrogel constructs}

To determine if FE-Pro labelled cells could be detected in vitro when seeded within synthetic 3D constructs, FE-Pro labelled hMSCs were imaged using MRI and confocal microscopy after seeding within PEG hydrogels. The hydrogel precursor solution was formed using $84 \%$ volume/volume (v/v) PEG-dimethylacrylate (PEGDMA: MW 750) (Sigma), 8\% v/v 2,2-Dimethoxy-2phenylacetophenone (Sigma) photoinitiator in 1-vinyl-2pyrrolidone (Sigma) crosslinker $(60 \mathrm{mg} / \mathrm{mL})$, and $8 \% \mathrm{v} / \mathrm{v}$ PBS. Two millilitres of this solution was placed into a well of a 6-well plate (Corning, Corning, NY, USA) and cells in solution were added to reach a concentration of $1 \times 10^{6}$ cells $/ \mathrm{mL}$. The plates were then exposed to 365 nanometre ultraviolet (UV) light (Uvitec, Cambridge, United Kingdom) for $30 \mathrm{~s}-5$ mins until gelation occurred. Of note, hydrogels seeded with FE-Pro labelled cells took longer (3-5 mins) to solidify under UV light compared to unlabelled cells and empty hydrogels (30s-2min).

\section{Ex vivo detection FE-Pro hMSCs in natural polymer fibrin gel}

In order to determine whether FE-Pro labelled cells could be detected within a natural polymer loaded into an animal model, labelled cells were imaged ex vivo within the IVD of excised rat tails. Labelled cells were loaded into a fibrin gel (Tisseel VH Fibrin Sealant, Baxter International Inc., Deerfield, IL, USA) at a concentration of $1 \times 10^{7}$ cells $/ \mathrm{mL}$, according to the manufacturer's instructions. Injection into the IVD was guided by the use of a fluoroscope (Xiscan C-arm Assembly, Xitec, Windsor Locks, CT, USA). A syringe needle was used to push the nucleus pulposus out of the IVD, and $50 \mu \mathrm{L}$ of the gel containing labelled cells was injected into the region. Based on previous retention studies, approximately $10 \mu \mathrm{L}$ is retained initially within the disc, yielding an estimate of $1 \times 10^{5}$ cells present following injection. As a control, an empty fibrin gel was injected into an adjacent disc within the rat tail. X-ray images (Faxitron X-ray, Wheeling, IL, USA) were obtained prior to and following injection, and the difference in disc height measured as an indicator that the gel containing labelled hMSCs was in fact injected into the IVD. All research involving animals was performed under approved guidelines established by the UCSF institutional animal care and use committee.

\section{MR imaging}

All MR imaging experiments were performed on a $3 \mathrm{~T}$ scanner (GE Medical Systems, Waukesha, WI, USA), and Mayo wrist coil (Mayo Clinic, Rochester, MN, USA). For in vitro imaging of cells in solution, cells at concentrations ranging from $1 \times 10^{4}-10^{6}$ cells $/ \mathrm{mL}$ were suspended in tubes containing $0.5 \mathrm{~mL}$ Ficoll $(1.077 \mathrm{~g} / \mathrm{mL}$ ) (Amersham Biosciences, Piscataway, NJ, USA). Ficoll was used to suspend the cells in order to maintain osmolarity and prevent cell settling. As a negative control, unlabelled hMSCs and an empty Ficoll solution were also imaged. For each concentration of labelled cells, and the unlabelled control, multiple samples $(\mathrm{n}=3)$ were prepared for in vitro imaging, and the results averaged. For cells labelled and seeded in hydrogels, the cross-linked gels were placed within the imaging tubes without Ficoll. For the hydrogel experiment controls, empty hydrogels were imaged, as well as hydrogels containing unlabelled hMSCs. Tubes were placed in a water bath and imaged at room temperature $\left(20^{\circ} \mathrm{C}\right)$. A spin-echo $(\mathrm{SE})$ sequence was used to determine $\mathrm{T}_{1}$ (Echo Time $(\mathrm{TE}) /$ Repetition Time $(\mathrm{TR})=12 / 60$ $1000 \mathrm{~ms})$ and $\mathrm{T}_{2}(\mathrm{TE} / \mathrm{TR}=12-300 / 4000 \mathrm{~ms})$ relaxation times. A gradient-echo (GRE) sequence was used to measure $\mathrm{T}_{2} *$ relaxation times using a $90^{\circ}$ flip angle, and $\mathrm{TE} / \mathrm{TR}=4-20 / 34 \mathrm{~ms}$. The field of view (FOV) used was $60 \times 60 \mathrm{~mm}$, and the in plane resolution of the single slice was $234 \mu \mathrm{m}$ on a side with a thickness of $9 \mathrm{~mm}$. Dedicated processing software written in Interactive Data Language (Research Systems, Boulder, CO) and MATLAB (Mathworks, Natick, MA, USA) was used to quantify $\mathrm{T}_{2}{ }^{*}$ relaxation times by performing a two parameter (initial signal-intensity (SI) and $\mathrm{T}_{2}{ }^{*}$ relaxation time) exponential fit of the experimentally obtained data points. This was done for each of the samples $(n=3)$, and the results averaged. In cases where FE-Pro labelling led to saturation of SI loss into the background noise, two different methods were used for fitting. In the first method, only the linear region on natural log plots of SI was used for curve fitting. In the second method, the images were converted to power images by squaring the magnitude images. These power images were then used for a three parameter fit, in which the initial SI, the $\mathrm{T}_{2} *$ relaxation time, and an additive noise term were optimized.

For ex vivo natural polymer detection of labelled cells within the excised rat tails, specimens were scanned coronally using a 3D multi-slice GRE sequence (TE/TR = $8 / 24 \mathrm{~ms}$ ). The in-plane resolution of the obtained images was $117 \mu \mathrm{m}$ on a side, with a slice thickness of $0.6 \mathrm{~mm}$, and a total imaging thickness of $14 \mathrm{~mm}$. The FOV for the region imaged was $60 \mathrm{~mm}$.

\section{Confocal microscopy}

FE-Pro labeled hMSCs seeded within PEG based hydrogels were also imaged using confocal microscopy. Following FE-Pro labelling, hMSCs were also labelled using CellTracker CMFDA (Invitrogen) according to the manufacturer's directions. These cells were then seeded within PEG based hydrogels and placed in 6-well plates. A stack of images was obtained throughout the entire volume of the hydrogel using a Nikon C1SI imaging system (Nikon USA, Melville, NY, USA) with a $488 \mathrm{~nm}$ excitation wavelength and a $30 \mathrm{~nm}$ pass-band centred at $515 \mathrm{~nm}$ (green). The imaging setup consisted of a water immersion lens with a $10 x$ objective and a 0.3 numerical aperture. The FOV for each slice in the stack was $615 \mu \mathrm{m}$ on a side, with a slice thickness of $5 \mu \mathrm{m}$, and total stack thickness of $205 \mu \mathrm{m}$. The stack of images was processed, and combined into a $3 \mathrm{D}$ volume using Nikon image processing software (NIS Elements AR 2.30). 
a)

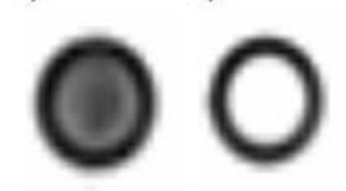

c)

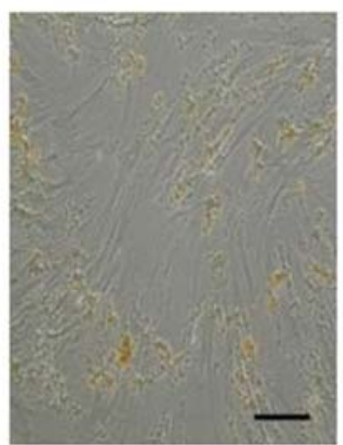

d)

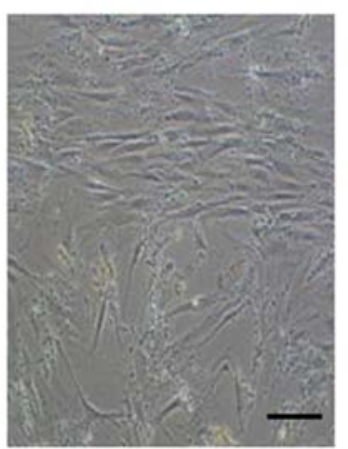

e)

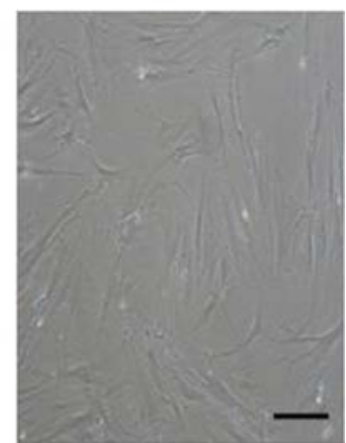

Figure 1: Iron oxide labelling of human mesenchymal stem cells: in vitro gradient echo (echo time/repetition time $=4 / 34 \mathrm{~ms})$ magnetic resonance image of (a) iron oxide labelled cells $\left(1 \times 10^{6}\right.$ cells $\left./ \mathrm{mL}\right)$; and (b) unlabeled cells $\left(1 \times 10^{6}\right.$ cells $\left./ \mathrm{mL}\right) ;(\mathbf{c})$ optical micrograph of labeled cells before heparin wash; (d) optical micrograph of labelled cells after heparin wash; (e) optical micrograph of unlabeled cells. (scale bar $=40 \mu \mathrm{m}$ )

\section{Cell viability}

To assess viability of cells following labelling, FE-Pro labelled and control hMSCs were plated at $70 \%$ confluence in 2-well Permanox chamber slides (Nalge Nunc International, Rochester, NY, USA). At days 0, 1, 2, 4, and 8 after labelling, hMSC viability was assessed with the Live/Dead Reduced Biohazard Viability/Cytotoxicity Kit (Invitrogen). Viability of unlabelled cells was also assessed as a control. Cells were incubated in a solution containing $2 \mu \mathrm{L}$ SYTO 10 green fluorescent nucleic acid stain, and $2 \mu \mathrm{L}$ ethidium homodimer- 2 in $1 \mathrm{~mL}$ of $(4-(2-$ hydroxyethyl)-1-piperazineethanesulfonic acid) buffered saline solution at room temperature in the dark for 30 minutes. Cells were then viewed with a fluorescent microscope and digital micrographs were obtained using both a fluorescein (Excitation/Emission: 494/517nm) and texas red filter (Excitation/Emission 528/617nm). Images were loaded into Adobe Photoshop (Adobe Systems, San Jose CA, USA), and using an overlaid grid live and dead cells were quantified through hand counts performed by lab personnel. The ratio of dead cells to live cells was recorded and used for statistical analysis.

\section{Statistical Analysis}

Statistical analysis of the obtained relaxation times was performed using an analysis of variance (ANOVA) test, followed by a Holm t-test to compare each of the labelled samples against the unlabeled control. For the cell viability data an ANOVA test on the ratio of dead cells to live cells for the 5 experimental groups and the single control group was used.

\section{Results}

\section{Effect of FE-Pro labelling on signal characteristics}

The results of the in vitro experiments indicate efficient cellular uptake of the contrast agent via FE-Pro complex formation, and the corresponding distinguishable SI loss of labelled cells on MRI images (Fig. 1). Labelled cells (Fig. 1a) show up considerably darker than unlabelled cells (Fig. 1b). Digital micrographs showing labelled cells before heparin wash (Fig. 1c), labelled cells after wash (Fig. 1d), and unlabelled cells (Fig. 1e) are also depicted. Plots of SI versus TR ( $\mathrm{T}_{1}$ measurements) and SI versus $\mathrm{TE}\left(\mathrm{T}_{2}\right.$, and $\mathrm{T}_{2} *$ measurements $)$ are depicted in Figure 2. On $\mathrm{T}_{1}$ weighted images (Fig. 2a), FE-Pro labelling does not appear to have an effect on SI, and labelled cells are not distinguishable from unlabelled cells. The effect of FE-Pro labelling is most pronounced on $\mathrm{T}_{2}{ }^{*}$ weighted GRE images (Fig. 2b). Cells labelled at the highest concentration of $1 \times 10^{6}$ cells $/ \mathrm{mL}$ show the greatest loss in SI, while labelled cells at a concentration of $1 \times 10^{4}-10^{5}$ cells $/ \mathrm{mL}$ also have lower SI values at low TEs compared to unlabelled hMSCs. On $\mathrm{T}_{2}$ weighted images (Fig. 2c), labelled cells appear to be distinguishable from unlabeled cells at longer TEs (70ms-300ms), but SI loss does not appear to vary with the concentration of labelled cells. Focusing on the $\mathrm{T}_{2}{ }^{*}$ weighted GRE images where SI loss is strongest, $\mathrm{T}_{2}{ }^{*}$ relaxation times obtained by using a two parameter fit are depicted in Figure 2d. Compared to the unlabelled control, there is a statistically significant difference $(\mathrm{p}<0.01)$ in the $\mathrm{T}_{2} *$ values for the labelled cells at $1 \times 10^{6}$ cells $/ \mathrm{mL}$. Figure 2e shows the $\mathrm{T}_{2}{ }^{*}$ results of the three parameter fit. Including noise in the fit yields a higher $\mathrm{T}_{2}{ }^{*}$ value for $1 \times 10^{6}$ cells $/ \mathrm{mL}$ as compared to the two parameter fit, but the difference between relaxation times for labelled and unlabelled cells at the highest concentration is still statistically significant $(\mathrm{p}<0.01)$.

\section{FE-Pro hMSCs in hydrogel constructs}

Seeding of labelled stem cells within the PEG based hydrogels resulted in in vitro detection via MRI (Figs. 3ac). Labelled cells within the hydrogel appeared as a hypointense region on the images obtained. Imaging of cells using confocal microscopy allowed detection of single cells throughout the volume of the hydrogel. A representative confocal microscopy volume image is shown in Figures 3d-e. Cells were stained using the CellTracker CMFDA kit, which stains only live cells. The positive stain indicates that the FE-Pro hMSCs were well distributed within the hydrogel, and that the FE-Pro hMSCs survived the labelling procedure and the hydrogel photo polymerization process. 

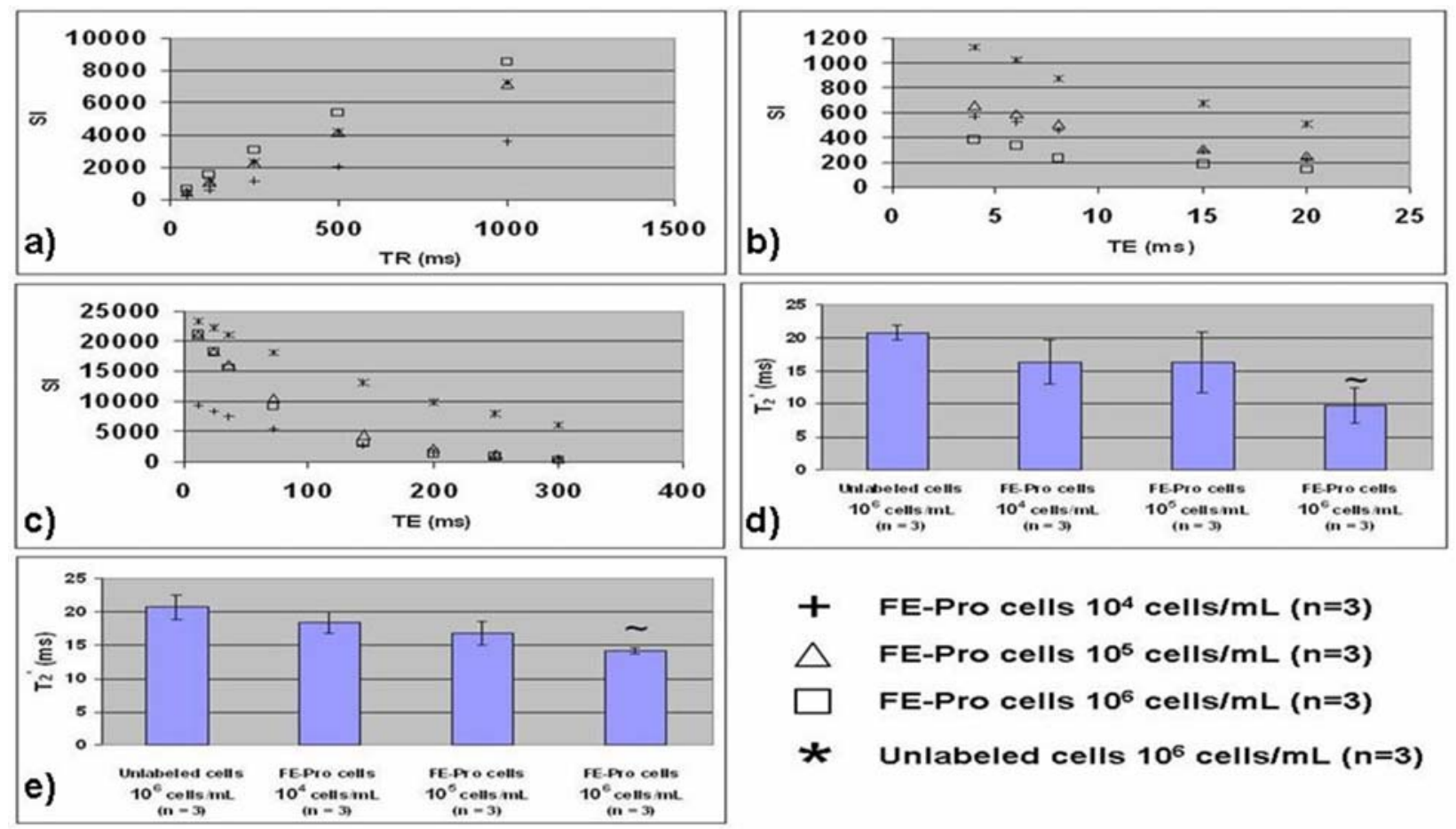

Figure 2: Signal Intensity (SI) and relaxation measurements: (a) SI vs. repetition time (TR) plot resulting from spin echo sequence. Labelled cells are indistinguishable; (b) SI vs. echo time (TE) plot resulting from gradient echo sequence. SI values are lower for labelled cells; (c) SI vs. TE plot resulting from spin echo sequence. Labelled cells are distinguishable from unlabelled cells at TEs above $70 \mathrm{~ms}$; (d) $\mathrm{T}_{2}{ }^{*}$ relaxation times for labelled and unlabelled cells obtained by fitting SI values to an exponential curve. For FE-Pro cells at $1 \times 10^{6}$ cells $/ \mathrm{mL}$ only TEs of 4, 6, and $8 \mathrm{~ms}$ were used for fitting to account for signal loss saturation. (e) $\mathrm{T}_{2}{ }^{*}$ relaxation times obtained by including a noise term in the exponential fit. ( $\mathrm{n}=\#$ of samples for each concentration; $\sim=$ statistical significance $p<.01$ compared to unlabelled cells)

a)

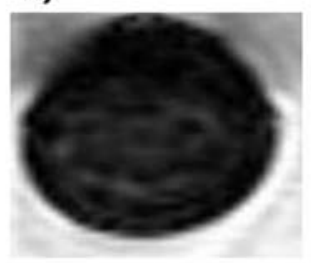

d)

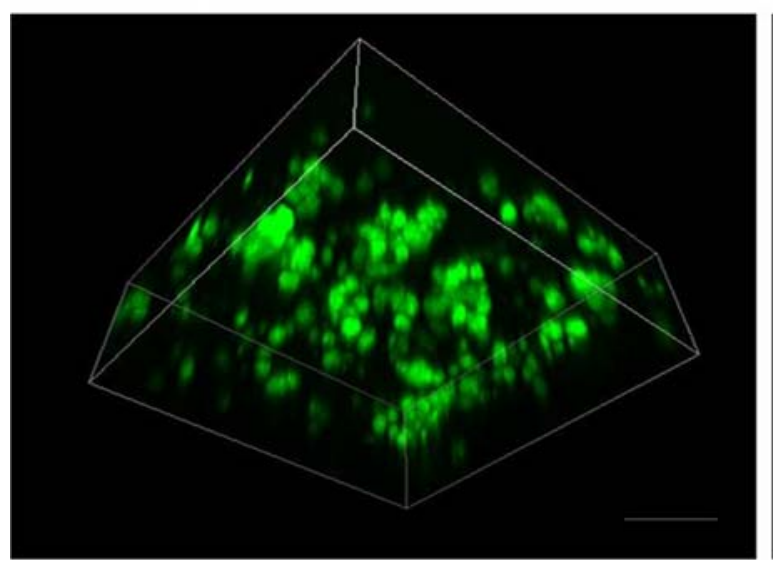

b)

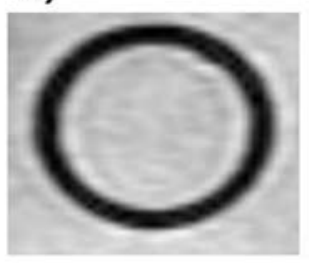

e) c)

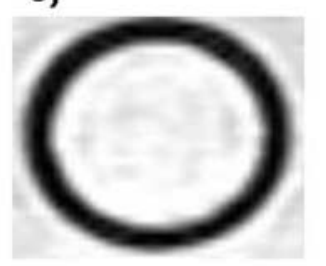

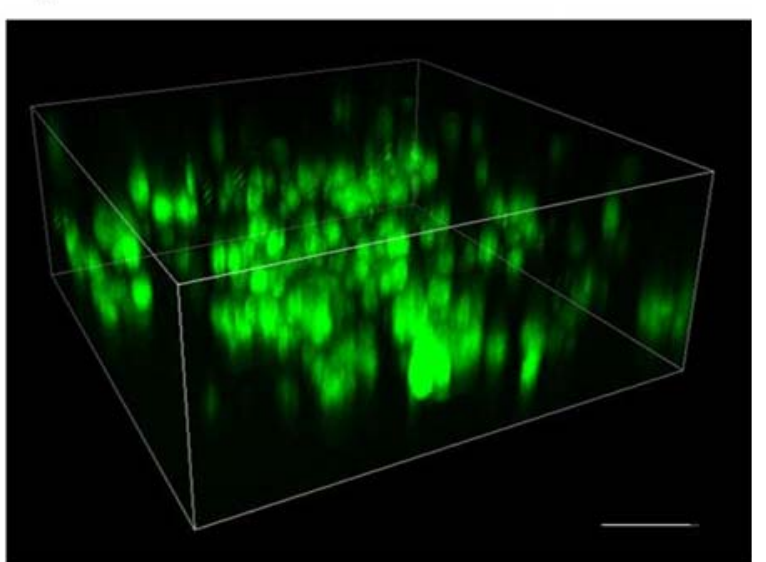

Figure 3: Magnetic resonance imaging and confocal microscopy of labelled cells within hydrogels: in vitro gradient echo (echo time/repetition time 6/34ms) magnetic resonance image of (a) stem cells labelled with the iron oxide complex seeded within the hydrogel ( $1 \times 10^{6}$ cells); (b) unlabeled stem cells ( $1 \times 10^{6}$ cells $)$ seeded within the hydrogel and; (c) hydrogel without cells. Labelled cells showed up as a hypointense region compared to unlabelled cells and the empty hydrogel; (d) \& (e) confocal microscopy volume rendered images of iron oxide labelled stem cells within a hydrogel. Positive green staining indicates the position of live cells following labelling and hydrogel polymerization. $($ scale bar $=100 \mu \mathrm{m})$ 


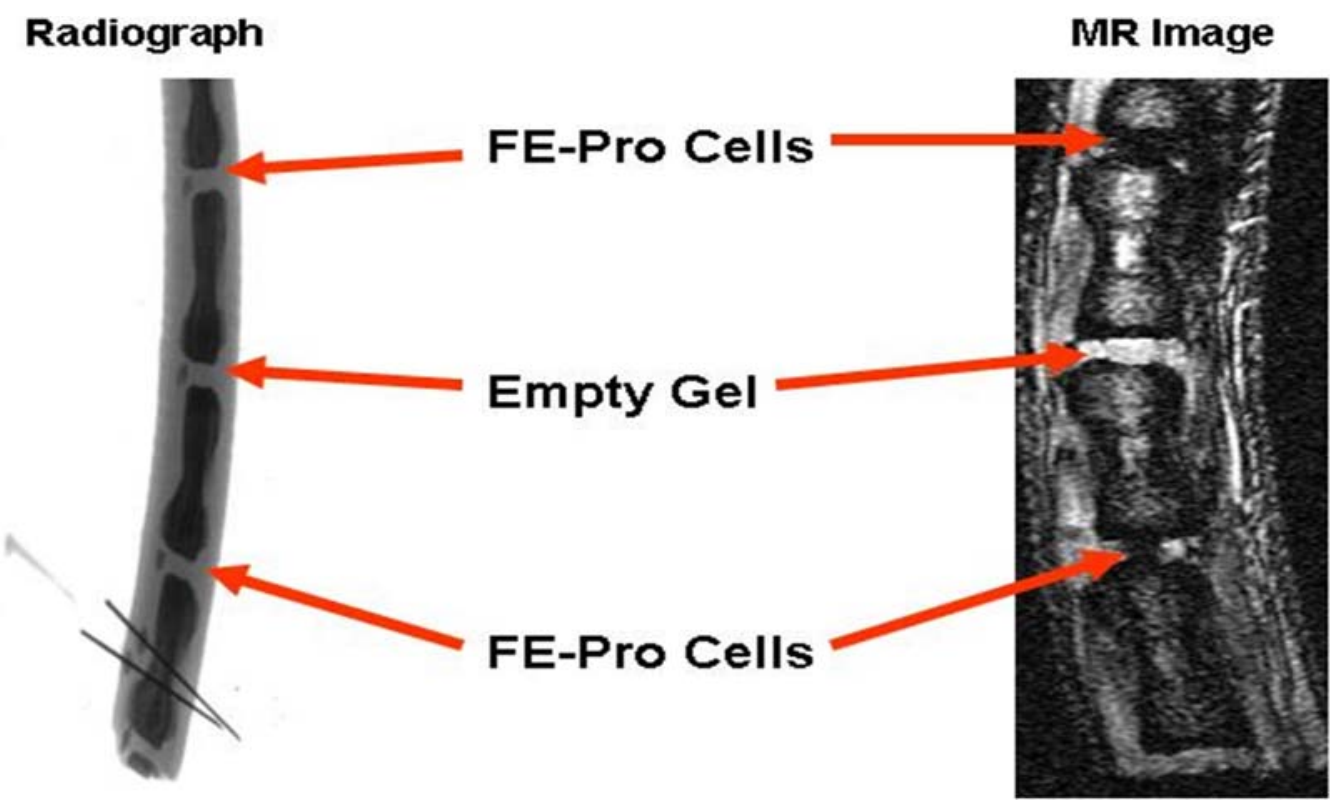

Figure 4: Ex vivo detection of iron oxide labelled cells within a rat model of disc degeneration: Rat discs were injected with a fibrin gel containing labelled cells $\left(1 \times 10^{7}\right.$ cells $\left./ \mathrm{mL}\right)$ and imaged using magnetic resonance imaging. Discs injected with labelled cells appear darker indicating a loss of signal on the image (right). For comparison, a disc was injected with fibrin gel alone, and appears brighter. A radiograph image (left) shows the sites of injection. Disc height was also increased following injection.

\section{Ex vivo MR detection of labelled cells}

In the rat model (Fig. 4), the signal loss due to FE-Pro labelling is clearly apparent in the injected discs. While the labelled cells in the disc appear black in the image, the disc injected with only fibrin gel is brighter. A radiograph image depicting the sites of injection is also shown. In addition, disc height following injection as measured on radiographs is increased (not shown) when compared to disc height prior to injection of the fibrin gel. This was used as an indicator that the cells seeded within the fibrin gel were in fact injected into the IVD.

\section{Cell viability}

The labelling of hMSCs with FE-Pro does not significantly impair cell viability. When compared against unlabeled hMSCs (control) the ratio of dead cells to live cells was not significantly different at day $0,1,2,4$ and 8 after labelling $(\mathrm{P}>0.25)$. Figure 5 shows the ratio of dead cells to live cells for the labelled groups at various days, as well as the unlabelled control group.

\section{Discussion}

The promise of utilizing stem cells to regenerate diseased or damaged tissue inherently requires an effective minimally invasive method for assessment. With MRI tracking, FE-Pro labelled cell populations appear as signal voids, and thus can be distinguished on MR images. Since MR imaging is non-invasive, the ability to track stem cells using this imaging modality is highly favourable. While MRI tracking of iron oxide labelled cells has been examined in the past, the application of stem cell labelling to monitor IVD regeneration has not been previously

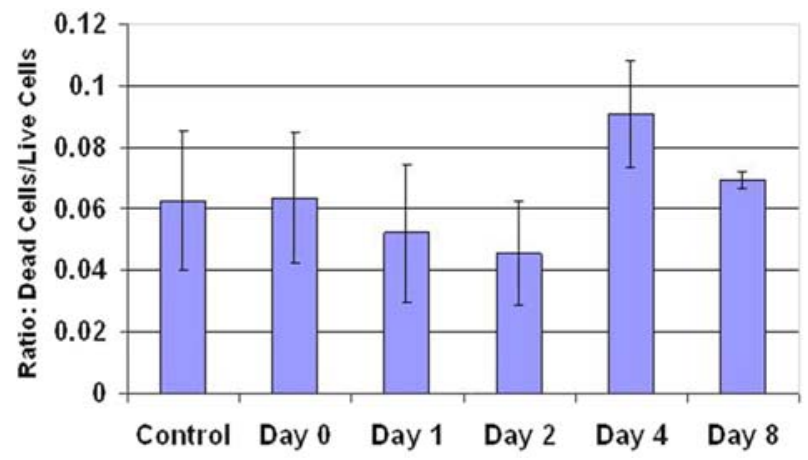

Figure 5: Effect of iron oxide labelling on cell viability: The ratio of dead cells to live cells between unlabelled controls, and labelled cells at day $0,1,2,4, \& 8$ after labelling was compared. There appears to be no significant difference in cell viability between labelled cells and unlabelled cells. Iron oxide labelling does not impair cell viability. (error bars correspond to mean \pm standard deviation)

investigated. Application of this technique to the IVD is important because the ability to track implanted hMSCs within the disc would be valuable as a means of noninvasively monitoring the repair and regeneration process. This would make it possible to determine the effectiveness of such treatment. As demonstrated by the results of this study, FE-Pro labelling and tracking using MRI has promise as a means for studying stem cell based regeneration of the IVD. Specifically, the results show that SI loss of labelled cells on MR images can be quantitatively characterized by measurements of standard MRI relaxation parameters. In addition, FE-Pro labelled cells can be distinguished from the surrounding environment when placed within synthetic scaffolds such as hydrogels, as well 
as within natural polymers implanted within native tissue. Lastly, cell viability of labelled cells does not appear to be affected by labelling with FE-Pro.

The SI loss and subsequent contrast of labelled cells is strongest on GRE images (Figure 1a-b). GRE pulse sequences are utilized because they emphasize differences in magnetic susceptibility between tissues. For this particular methodology, stem cells labelled with FE-Pro have increased susceptibility due to the highly magnetic iron oxide particles. In addition, GRE imaging allows for imaging at short TEs, where differences in SI and associated contrast are the greatest for iron oxide labelled cells. These characteristics of GRE pulse sequences allow for the strong contrast of iron oxide labelled cells and their detection on the resulting images. Based on the results of the quantitative analysis, cells at a concentration of $1 \times 10^{6}$ cells $/ \mathrm{mL}$ are distinguishable from unlabelled cells $\left(1 \times 10^{6}\right.$ cells $/ \mathrm{mL}$ ) in vitro, with a statistically significant difference in $\mathrm{T}_{2}{ }^{*}$ values when both the two and three parameter fit are used. Clinically, the number of cells implanted for therapeutic applications will depend on the particular site and tissue of interest, but it is important that the size of the transplanted cell population is within the detection limits of MRI. Miller and Joseph (1993) report that fitting of the associated power images with an additive noise term generally causes a decrease in the measured relaxation time when compared to a two parameter fitting of the magnitude images. The fact that this differs from the results obtained in this study for labelled cells at the highest concentration is presumably because the $\mathrm{T}_{2}{ }^{*}$ values measured here are very short. Thus the effect discussed by Miller and Joseph (1993) is less dominant in this situation. For the other concentrations of labelled cells, and the unlabelled cells, the SI plots indicate that the signal loss is not saturated into the noise region, which leads to similar $\mathrm{T}_{2}{ }^{*}$ values using both the two and three parameter fit.

Stem cell populations are often implanted within the body using a scaffold to create an environment that is conducive to cell attachment, proliferation, required gene expression, and formation of new functional tissue (O'Halloran and Pandit, 2007). Ideally, the scaffold mimics the in vivo environment of the implanted cells for a defined period of time, until the implanted cells integrate into the surrounding native tissue. Presently, a variety of synthetic and natural polymers have been utilized for this purpose (Raghunath et al., 2007). MRI detection of labelled cells specifically within 3D scaffolds is an important consideration, because each type of polymer will have its own MRI signal characteristics and associated SI based on its material composition. Thus, the signal characteristics of the FE-Pro labelled cells seeded within the 3D scaffold must be iron oxide dominated with sufficient SI loss and associated contrast to allow for adequate detection. Among synthetic polymers, the use of hydrogels as 3D scaffolds for cell implantation has been well documented (Brandl et al., 2007). We report here the in vitro detection of FE-Pro labelled hMSCs seeded within hydrogels using MRI. Labelled cells within the synthetic PEG hydrogels were also stained and imaged using confocal microscopy, illustrating the FE-Pro labelled stem cells can be enclosed in a scaffold, and remain both live and viable. Currently, resolution limits preclude imaging of single cells using standard MRI machines. However, the ability to track a transplanted population of labelled stem cells longitudinally represents a significant step in the assessment of stem cell based therapies. Furthermore, as the resolution of clinical MRI continues to improve, the ability to image single cells should be possible in the foreseeable future. Currently, Hinds et al. (2003) report the ability to image single cells in vitro using an 11.7 Tesla spectrometer.

In addition, we have demonstrated the ex vivo detection of a transplanted population of labelled hMSCs seeded within a fibrin based gel, a well characterized natural polymer utilized in tissue regeneration (Peretti et al., 2000; Westreich et al., 2004). As expected the discs implanted with FE-Pro labelled cells appear as a hypointense region, and so are detectable by means of MRI (Figure 4). These results are similar to those reported by Sykova et al. (2006) who were able to detect iron oxide labelled rat stem cells when implanted in vivo within the spinal cord of injured rats. MRI has already been extensively used to image the IVD (Boos et al., 1994; Masaryk et al., 1988), and techniques have been developed to classify disc degeneration based on MR images (Pearce et al., 1991; Pfirrmann et al., 2001; Raininko et al., 1995; Schiebler et al., 1991; Tertti et al., 1991; Thompson et al., 1990). Consequently, as stem cell based techniques for IVD regeneration are developed, utilizing MRI to image the disc and monitor therapy seems to be a feasible and promising endeavour.

Labelling of hMSCs with FE-Pro does not appear to affect cell viability. These results are in agreement with those obtained by Arbab et al. (2004b) who reported no increased cell death following labelling, and no reduction in proliferation capacity. As an indication that cells were in fact labelled, Arbab et al. (2004b) report 100\% labelling efficiency, as determined by Prussian Blue Staining and manual counting of cells prepared on microscope slides. In looking to future applications, both components of the FE-Pro complex as well as the heparin used for washing are FDA approved, and so are not expected to have toxic effects when utilized in vivo.

While this study utilized FE-Pro labelling to provide negative contrast via SI loss, it is also possible to generate positive contrast with iron oxide labelling. Cunningham et al. (2005) demonstrated the ability to label cells with SPIOs and use spectrally selective pulses to excite the off resonance water surrounding the labelled cells. This allowed for positive contrast of the labelled cells compared to the surroundings, as well as a linear correlation between the SI and the number of cells. Based on these preliminary results, future studies should consider the ability to detect FE-Pro labelled hMSCs using positive contrast MR imaging sequences, and the effects on detection within the surrounding tissue of the IVD. This can be done both qualitatively using obtained images and quantitatively via MR relaxation parameters. Since negative contrast imaging is limited in its ability to distinguish between the presence of labelled cells and an inherent signal void in the image, 
the use of positive contrast to confirm the presence of labelled cells would be beneficial. In principle it is possible to use a combination of positive and negative contrast imaging to track transplanted labelled cells simply by varying the pulse sequence used during each scan. As a result, it is expected that positive contrast imaging could be used for initial detection following transplantation of labelled cells within the IVD, followed by high resolution negative contrast imaging to image the cells within the surrounding tissue once they have been detected.

In conclusion, this study demonstrated that the established technique of labelling with iron oxides and MR imaging appears feasible as a methodology for monitoring stem cell based regeneration of the IVD. As stem cell based therapies continue to emerge as treatment methods for diseased or damaged tissues, the use of this technique for non-invasive assessment shows promise. With the demonstrated in vitro and ex vivo results presented here, future studies should include in vivo longitudinal studies of stem cell tracking by means of MRI. As this technique continues to show promising results, its clinical application to treatment of IVD degeneration and a variety of other disease conditions is expected.

\section{Acknowledgements}

All confocal images were acquired at the Nikon Imaging Centre at UCSF/QB3 with the help of Dr. Kurt Thorn. Ex vivo implantation of labelled cells within the rat IVD was performed by Ms. Anne Kim within the lab of Dr. Jeffrey Lotz (UCSF Dept. of Orthopaedic Surgery). This research was supported by NIHRO1-AG17762

\section{References}

Arbab AS, Jordan EK, Wilson LB, Yocum GT, Lewis BK, Frank JA (2004a) In vivo trafficking and targeted delivery of magnetically labeled stem cells. Hum Gene Ther 15: 351-360.

Arbab AS, Yocum GT, Kalish H, Jordan EK, Anderson SA, Khakoo AY, Read EJ, Frank JA (2004b) Efficient magnetic cell labeling with protamine sulfate complexed to ferumoxides for cellular MRI. Blood 104: 1217-1223.

Arbab AS, Yocum GT, Rad AM, Khakoo AY, Fellowes V, Read EJ, Frank JA (2005) Labeling of cells with ferumoxides-protamine sulfate complexes does not inhibit function or differentiation capacity of hematopoietic or mesenchymal stem cells. NMR Biomed 18: 553-559.

Battie MC, Videman T (2006) Lumbar disc degeneration: epidemiology and genetics. J Bone Joint Surg Am 88 Suppl 2: 3-9.

Beadle OA (1931) The intervertebral discs: observations on their normal and morbid anatomy in relation to certain spinal deformities. Medical Research Council Special Report Series 161.

Boos N, Wallin A, Schmucker T, Aebi M, Boesch C (1994) Quantitative MR imaging of lumbar intervertebral disc and vertebral bodies: methodology, reproducibility, and preliminary results. Magn Reson Imaging 12: 577587.

Boos N, Weissbach S, Rohrbach H, Weiler C, Spratt KF, Nerlich AG (2002) Classification of age-related changes in lumbar intervertebral discs: 2002 Volvo Award in basic science. Spine 27: 2631-2644.

Brandl F, Sommer F, Goepferich A (2007) Rational design of hydrogels for tissue engineering: impact of physical factors on cell behavior. Biomaterials 28: 134146.

Bulte JW, Douglas T, Witwer B, Zhang SC, Strable E, Lewis BK, Zywicke H, Miller B, van Gelderen P, Moskowitz BM, Duncan ID, Frank JA (2001) Magnetodendrimers allow endosomal magnetic labeling and in vivo tracking of stem cells. Nat Biotechnol 19:11411147.

Crevensten G, Walsh AJ, Ananthakrishnan D, Page P, Wahba GM, Lotz JC, Berven S (2004) Intervertebral disc cell therapy for regeneration: mesenchymal stem cell implantation in rat intervertebral discs. Ann Biomed Eng 32: $430-434$.

Cunningham CH, Arai T, Yang PC, McConnell MV, Pauly JM, Conolly SM (2005) Positive contrast magnetic resonance imaging of cells labeled with magnetic nanoparticles. Magn Reson Med 53: 999-1005.

Elisseeff J, Ferran A, Hwang S, Varghese S, Zhang Z (2006) The role of biomaterials in stem cell differentiation: applications in the musculoskeletal system. Stem Cells Dev 15: 295-303.

Frank JA, Miller BR, Arbab AS, Zywicke HA, Jordan EK, Lewis BK, Bryant LH, Jr., Bulte JW (2003) Clinically applicable labeling of mammalian and stem cells by combining superparamagnetic iron oxides and transfection agents. Radiology 228: 480-487.

Hinds KA, Hill JM, Shapiro EM, Laukkanen MO, Silva AC, Combs CA, Varney TR, Balaban RS, Koretsky AP, Dunbar CE (2003) Highly efficient endosomal labeling of progenitor and stem cells with large magnetic particles allows magnetic resonance imaging of single cells. Blood 102: $867-872$.

Hoehn M, Kustermann E, Blunk J, Wiedermann D, Trapp T, Wecker S, Focking M, Arnold H, Hescheler J, Fleischmann BK, Schwindt W, Buhrle C (2002) Monitoring of implanted stem cell migration in vivo: a highly resolved in vivo magnetic resonance imaging investigation of experimental stroke in rat. Proc Natl Acad Sci USA 99: 16267-16272.

Kalish H, Arbab AS, Miller BR, Lewis BK, Zywicke HA, Bulte JW, Bryant LH, Jr., Frank JA (2003) Combination of transfection agents and magnetic resonance contrast agents for cellular imaging: relationship between relaxivities, electrostatic forces, and chemical composition. Magn Reson Med 50: 275-282.

Lagasse E, Connors H, Al-Dhalimy M, Reitsma M, Dohse M, Osborne L, Wang X, Finegold M, Weissman IL, Grompe M (2000) Purified hematopoietic stem cells can differentiate into hepatocytes in vivo. Nat Med 6: 12291234.

Leung VY, Chan D, Cheung KM (2006) Regeneration of intervertebral disc by mesenchymal stem cells: 
potentials, limitations, and future direction. Eur Spine J 15 Suppl 3: S406-413.

Masaryk TJ, Ross JS, Modic MT, Boumphrey F, Bohlman H, Wilber G (1988) High-resolution MR imaging of sequestered lumbar intervertebral disks. AJR Am J Roentgenol 150: 1155-1162.

Miller AJ, Joseph PM (1993) The use of power images to perform quantitative analysis on low SNR MR images. Magn Reson Imaging 11: 1051-1056.

O’Halloran D M, Pandit AS (2007) Tissue-engineering approach to regenerating the intervertebral disc. Tissue Eng 13: 1927-1954.

Orlic D, Kajstura J, Chimenti S, Bodine DM, Leri A, Anversa P (2001) Transplanted adult bone marrow cells repair myocardial infarcts in mice. Ann N Y Acad Sci 938: 221-229; discussion 229-230.

Orlic D, Kajstura J, Chimenti S, Bodine DM, Leri A, Anversa P (2003) Bone marrow stem cells regenerate infarcted myocardium. Pediatr Transplant 7 Suppl 3: 8688.

Pearce RH, Thompson JP, Bebault GM, Flak B (1991) Magnetic resonance imaging reflects the chemical changes of aging degeneration in the human intervertebral disk. J Rheumatol Suppl 27: 42-43.

Peretti GM, Randolph MA, Villa MT, Buragas MS, Yaremchuk MJ (2000) Cell-based tissue-engineered allogeneic implant for cartilage repair. Tissue Eng 6: 567576.

Pfirrmann CW, Metzdorf A, Zanetti M, Hodler J, Boos N (2001) Magnetic resonance classification of lumbar intervertebral disc degeneration. Spine 26: 1873-1878.

Raghunath J, Rollo J, Sales KM, Butler PE, Seifalian AM (2007) Biomaterials and scaffold design: key to tissueengineering cartilage. Biotechnol Appl Biochem 46: 73 84.

Raininko R, Manninen H, Battie MC, Gibbons LE, Gill K, Fisher LD (1995) Observer variability in the assessment of disc degeneration on magnetic resonance images of the lumbar and thoracic spine. Spine 20: 1029-1035.

Sakai D, Mochida J, Iwashina T, Hiyama A, Omi H, Imai M, Nakai T, Ando K, Hotta T (2006) Regenerative effects of transplanting mesenchymal stem cells embedded in atelocollagen to the degenerated intervertebral disc. Biomaterials 27:335-345.

Sakai D, Mochida J, Yamamoto Y, Nomura T, Okuma M, Nishimura K, Nakai T, Ando K, Hotta T (2003) Transplantation of mesenchymal stem cells embedded in Atelocollagen gel to the intervertebral disc: a potential therapeutic model for disc degeneration. Biomaterials 24: 3531-3541.

Sakai D, Mochida J, Iwashina T, Watanabe T, Nakai T, Ando K, Hotta T (2005) Differentiation of mesenchymal stem cells transplanted to a rabbit degenerative disc model: potential and limitations for stem cell therapy in disc regeneration. Spine 30: 2379-2387.

Schiebler ML, Camerino VJ, Fallon MD, Zlatkin MB, Grenier N, Kressel HY (1991) In vivo and ex vivo magnetic resonance imaging evaluation of early disc degeneration with histopathologic correlation. Spine 16: 635-640.
Sykova E, Jendelova P, Urdzikova L, Lesny P, Hejcl A (2006) Bone marrow stem cells and polymer hydrogels two strategies for spinal cord injury repair. Cell Mol Neurobiol 26: 1113-1129.

Takagi Y, Nishimura M, Morizane A, Takahashi J, Nozaki K, Hayashi J, Hashimoto N (2005) Survival and differentiation of neural progenitor cells derived from embryonic stem cells and transplanted into ischemic brain. J Neurosurg 103: 304-310.

Takahashi T, Kalka C, Masuda H, Chen D, Silver M, Kearney M, Magner M, Isner JM, Asahara T (1999) Ischemia- and cytokine-induced mobilization of bone marrow-derived endothelial progenitor cells for neovascularization. Nat Med 5: 434-438.

Tertti M, Paajanen H, Laato M, Aho H, Komu M, Kormano M (1991) Disc degeneration in magnetic resonance imaging. A comparative biochemical, histologic, and radiologic study in cadaver spines. Spine 16: 629-634.

Thompson JP, Pearce RH, Schechter MT, Adams ME, Tsang IK, Bishop PB (1990) Preliminary evaluation of a scheme for grading the gross morphology of the human intervertebral disc. Spine 15: 411-415.

Urban JP, Winlove CP (2007) Pathophysiology of the intervertebral disc and the challenges for MRI. J Magn Reson Imaging 25: 419-432.

Westreich R, Kaufman M, Gannon P, Lawson W (2004) Validating the subcutaneous model of injectable autologous cartilage using a fibrin glue scaffold. Laryngoscope 114: 2154-2160.

Zhang YG, Guo X, Xu P, Kang LL, Li J (2005) Bone mesenchymal stem cells transplanted into rabbit intervertebral discs can increase proteoglycans. Clin Orthop Relat Res 430: 219-226.

\section{Web Reference}

1.http://wwwp.medtronic.com/Newsroom/ LinkedItemDetails.do?itemId=1101756222541 \&itemType=fact_sheet\&lang=en_US [17-07-2007].

\section{Discussion with Reviewer}

G. Beaudoin: Why was it chosen to fit a 3-parameter equation on 3 data points? It would be better off to keep all the data points and let the fit deal with the additive noise. The $\mathrm{T}_{2} *$ error should be normalized with a factor $\mathrm{S}=\operatorname{sqrt}\left(\mathrm{Xi}^{2} / \mathrm{NDF}\right)$ for a better estimation of the error (ref. AH Rosenfeld (1975), Ann Rev Nucl Sci 25: 555).

Authors: The fitting of the three parameters (including noise) was done by fitting data for the five experimentally obtained data points (corresponding to TE values of 4,6,8, $15, \& 20 \mathrm{~ms}$ ) as shown in Fig. 2e. All data points were kept for the noise fitting as the reviewer suggests should be done. This fitting was done for 3 different tubes containing $1 \times 10^{6}$ cells $/ \mathrm{mL}$. Thus the mean and standard deviation are based on fitting for 3 separate tubes, and not for the standard error of the $\mathrm{T}_{2} *$ fitting. 